\title{
Dermatosis neutrofílica de las palmas, una presentación y asociación poco frecuente
}

\author{
Brayan Alzate Hernandez; ; Camilo Parodi²; Alexander Velandia3; Adriana Marín; \\ Mariam Rolón ${ }^{5}$
}

\section{RESUMEN}

La dermatosis neutrofílica acral (síndrome de Sweet localizado) fue descrita inicialmente como una manifestación cutánea de predominio en el dorso de las manos, con características histopatológicas de una vasculitis leucocitoclástica. Posteriormente se describió la presencia de neutrófilos y los cambios paraclínicos de una dermatosis neutrofílica localizada. Esta entidad se asocia a la presencia de infecciones, enfermedades autoinmunitarias, fármacos y neoplasias. Reportamos el caso de una mujer de 69 años con antecedente de carcinoma papilar de tiroides con nódulos dolorosos en palmas compatibles con síndrome de Sweet localizado.

PALABRAS CLAVE: Dermatosis de las manos; Neutrofilos; Síndrome de Sweet.

\section{NEUTROPHILIC DERMATOSIS OF THE PALMS, AN UNCOMMON PRESENTATION AND ASSOCIATION}

\section{SUMMARY}

Acral neutrophilic dermatosis (localized Sweet syndrome) was described as a predominantly cutaneous manifestation on the back of the hands, with histopathological characteristics of a leukocytoclastic vasculitis. The presence of neutrophils and the paraclinical changes of a localized neutrophilic dermatosis were subsequently described. This entity is associated with the presence of infections, autoimmune diseases, drugs, and neoplasms. We report the case of a 69-year-old woman with a history of papillary thyroid carcinoma with painful nodules on the palms compatible with localized Sweet's syndrome.

KEY WORDS: Hand dermatoses; Sweet syndrome; Neutrophils.

1. Residente de especialización en Dermatología, Universidad El Bosque. ORCID https://orcid.org/oooo-0001-6509-4405

2. Residente de especialización en Dermatología, Universidad El Bosque. ORCID https://orcid.org/oooo-ooo2-7576-1205

3. Residente de especialización en Dermatología, Universidad El Bosque. ORCID https://orcid.org/oooo-ooo2-4851-2481

4. Dermatóloga, Hospital Simón Bolívar. Docente titular, Universidad El Bosque. ORCID https://orcid.org/oooo-0003-1410-1809

5. Dermatóloga, dermatopatóloga, Hospital Simón Bolívar. Docente, Universidad El Bosque.

ORCID https://orcid.org/0000-0003-0322-3042

Correspondencia: brayank6@hotmail.com; email: brayank6@hotmail.com

Recibido: 11/10/19; aceptado: 20/05/20

Cómo citar: Alzate-Hernandez B; Parodi C; Velandia A; Marín A; Roló M. Dermatosis neutrofílica de las palmas, una presentación y asociación poco frecuente. Rev. Asoc Colomb Dermatol. Vol 28(3): julio - septiembre, 2020, 250-253.

DOI: https://doi.org/10.29176/2590843X.1534

Financiación: ninguna, conflictos de interés: ninguno 


\section{REPORTE DE CASO}

Paciente femenina de 69 años, que ingresa al Hospital Simón Bolívar de Bogotá con antecedente de carcinoma papilar de tiroides desde hace 2 años, con metástasis a pulmón y a glándula suprarrenal, y que requirió pleurectomía y toracotomía como parte del manejo de su cuadro de dificultad respiratoria, secundario al compromiso metastásico.

Durante la hospitalización, la paciente presenta picos febriles cuantificados de $390 \mathrm{C}$ sostenidos durante 2 días. El hemograma mostró leucocitosis con predominio de neutrófilos, con proteína C-reactiva y velocidad de sedimentación globular elevadas.

El examen físico evidenció nódulos dolorosos de $1 \mathrm{~cm}$ y placas eritematoedematosas que ocupaban toda la superficie palmar bilateral (figura 1). Se descartaron otros focos de infección (urinario, pulmonar y gastrointestinal) que explicaran el cuadro febril, por lo cual se decidió realizar biopsia de una de las lesiones del borde radial palmar. El estudio histopatológico evidenció denso infiltrado de neutrófilos en dermis, con marcado edema y sin presencia de vasculitis (figura 2). Se realizó el diagnóstico de síndrome de Sweet localizado, de presentación palmar bilateral, y se decidió iniciar el manejo con prednisolona por vía oral, en dosis de $0,5 \mathrm{mg} / \mathrm{kg} / \mathrm{d}$, durante 10 días, tratamiento con el cual hubo resolución total de las lesiones descritas y mejoría del dolor referido por la paciente. Posteriormente se continuó con los controles por oncología para el manejo de su enfermedad de base, sin recurrencia de esta dermatosis.

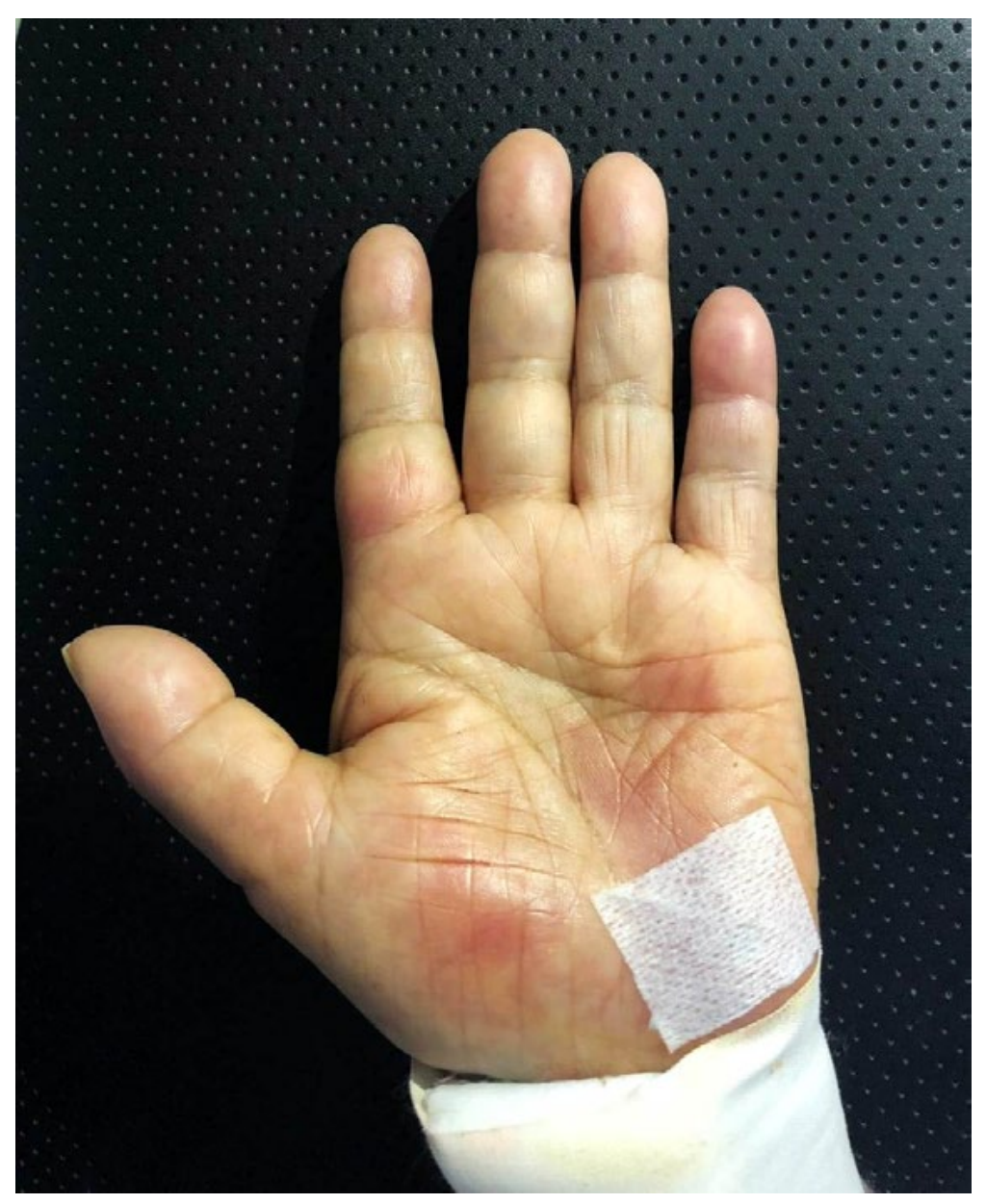

Figura 1. En la región palmar bilateral presentó nódulos dolorosos de $1 \mathrm{~cm}$ y placas eritematoedematosas que ocupaban toda la superficie. 


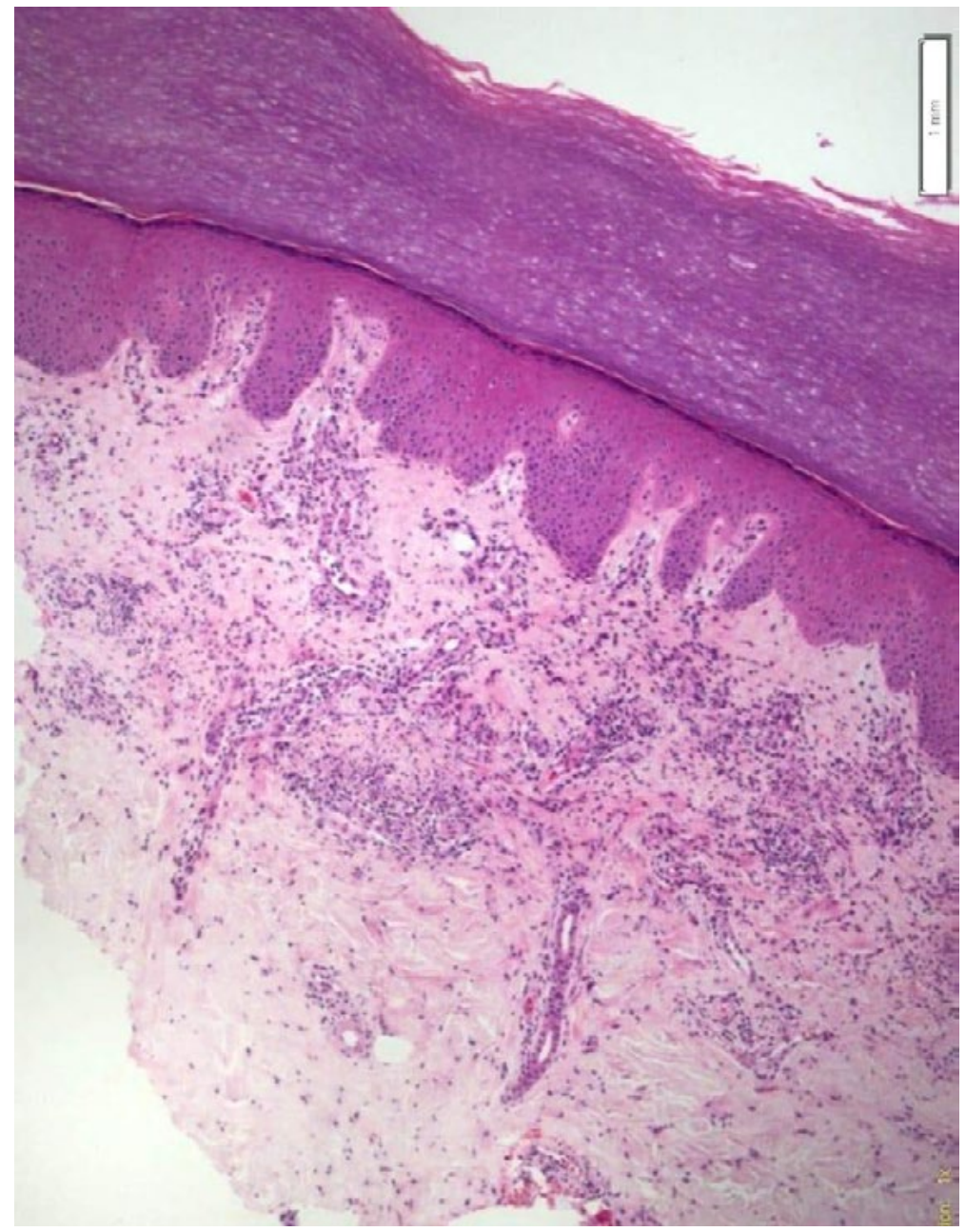

Figura 2. El estudio histopatológico evidenció denso infiltrado de neutrófilos en la dermis, con marcado edema y sin presencia de vasculitis.

\section{DISCUSIÓN}

La dermatosis neutrofílica de las manos fue descrita inicialmente como vasculitis pustulosa de las manos por Strutton y colaboradores en 1995, quienes encontraron en 6 pacientes lesiones que ocupaban el dorso de las manos con apariencia similar a un síndrome de Sweet y que histopatológicamente representaban una vasculitis leucocitoclástica. En el año 2000, Galaria describió casos similares, los cuales histopatológicamente no correspondieron a vasculitis, por lo que propuso el término dermatosis neutrofílica del dorso de las manos, el cual correspondería a una forma localizada del síndrome de Sweet ${ }^{(1-5)}$.
Esta es una patología poco frecuente. Aproximadamente se han reportado 100 casos en la literatura, con etiología no clara y con múltiples asociaciones como lo son las infecciones, principalmente del tracto respiratorio, la infección por hepatitis C, la enfermedad inflamatoria intestinal, las enfermedades autoinmunitarias, las inducidas por fármacos, las vacunas y las neoplasias. Las neoplasias más comunes son de origen hematológico (leucemias y síndromes mielodisplásicos) ${ }^{(2)}$. Es inusual la asociación a carcinoma papilar de tiroides, como lo reportamos en nuestro caso.

Clínicamente la localización más frecuente es el dorso de las manos, en forma de pústulas y pápulas ulce- 


\section{Puntos clave}

- La dermatosis neutrofílica de las manos corresponde a una forma localizada del síndrome de Sweet.

- Puede tener asociaciones con infecciones, enfermedad inflamatoria intestinal, enfermedades autoinmunitarias, fármacos, vacunas y neoplasias.

- La localización más frecuente es en el dorso de las manos, en forma de pústulas, pápulas ulceradas y placas eritematoedematosas.

- La primera línea de tratamiento son los corticosteroides orales.

radas, pero también se han descrito placas eritematoedematosas. La forma palmar es una variante rara y usualmente se presenta en forma de parches y ampollas; es infrecuente la presencia de nódulos dolorosos, como en el caso descrito ${ }^{(5)}$.

En la histopatología se observa un infiltrado dérmico denso de neutrófilos con importante edema subepidérmico, sin evidencia de vasculitis, aunque se han descrito algunos casos de vasculitis asociada; esta última explicada como un evento secundario relacionado con la intensidad del infiltrado de neutrófilos ${ }^{(3)}$.

Para el diagnóstico del síndrome de Sweet localizado, el uso de exámenes de laboratorio es de baja utilidad, ya que solo el $10 \%$ de los casos presenta leucocitosis con predominio de neutrófilos y reactantes de fase aguda elevados; sin embargo, en el caso de la paciente, estos fueron positivos, como suele presentarse en la forma convencional de síndrome de Sweet, donde sí es frecuente encontrar fiebre, leucocitosis con neutrofilia y lesiones cutáneas típicas. Es importante la búsqueda activa de las neoplasias asociadas, ya que en la mayoría de los casos estas pueden explicar la manifestación cutánea.

El diagnóstico diferencial se debe hacer con el grupo de dermatosis neutrofílicas, como el pioderma gangrenoso y el eritema elevatum diutinum, así como con procesos vasculíticos acrales, linfomas y artritis ${ }^{(4)}$.

El tratamiento de elección, al igual que las formas clásicas del síndrome de Sweet, es la administración de corticosteroides orales como terapia de primera línea. También se ha descrito el uso de dapsona, ciclosporina, metotrexato, colchicina, minociclina, tetraciclina y yoduro de potasio. El pronóstico y la recurrencia dependen del control de la enfermedad subyacente y/o de los desencadenantes ${ }^{(2-5)}$.

\section{CONCLUSIÓN}

Presentamos el caso de una mujer con diagnóstico de síndrome de Sweet localizado, una dermatosis poco frecuente, con presentación clínica atípica dada por la presencia de nódulos y localización palmar, además asociada a carcinoma papilar de tiroides, una neoplasia no hematológica como convencionalmente se observa en esta dermatosis. El tratamiento de elección son los corticoides sistémicos, con los cuales se logra una buena respuesta clínica.

\section{REFERENCIAS}

1. Costa-Silva M, Pedrosa A, Azevedo F, Mota A. Neutrophilic dermatosis of the dorsal hands: a restrictive designation for an acral entity. Acta Dermatovenerol Alp Pannonica Adriat. 2016;25(4):85-6. doi: 10.15570/actaapa.2016.25

2. Ramos FS, Ferreira FR, de Oliveira FM, Lanzoni M. Neutrophilic dermatosis of the dorsal hands: response to dapsone monotherapy. An Bras Dermatol. 2018;93(5):730-2. doi: 10.1590/abd18064841.20187488

3. Kaur S, Gupta D, Garg B, Sood N. Neutrophilic dermatosis of dorsal hands. Indian Dermatol Online J. 2015;6(1):42-5. doi: 10.4103/2229-5178.148940

4. Behrangi E, Rasi A, Attar B, Azizian Z. Neutrophilic Dermatosis of Dorsal Hands and Legs. Arch Iran Med. 2016;19(12):879-81.

5. Cheng AMY, Cheng HS, Smith BJ, Stewart DA. Neutrophilic Dermatosis of the Hands: A Review of 17 Cases. J Hand Surg Am. 2018;43(2):185.e1-e5. doi: 10.1016/j.jhsa.2017.08.027 